
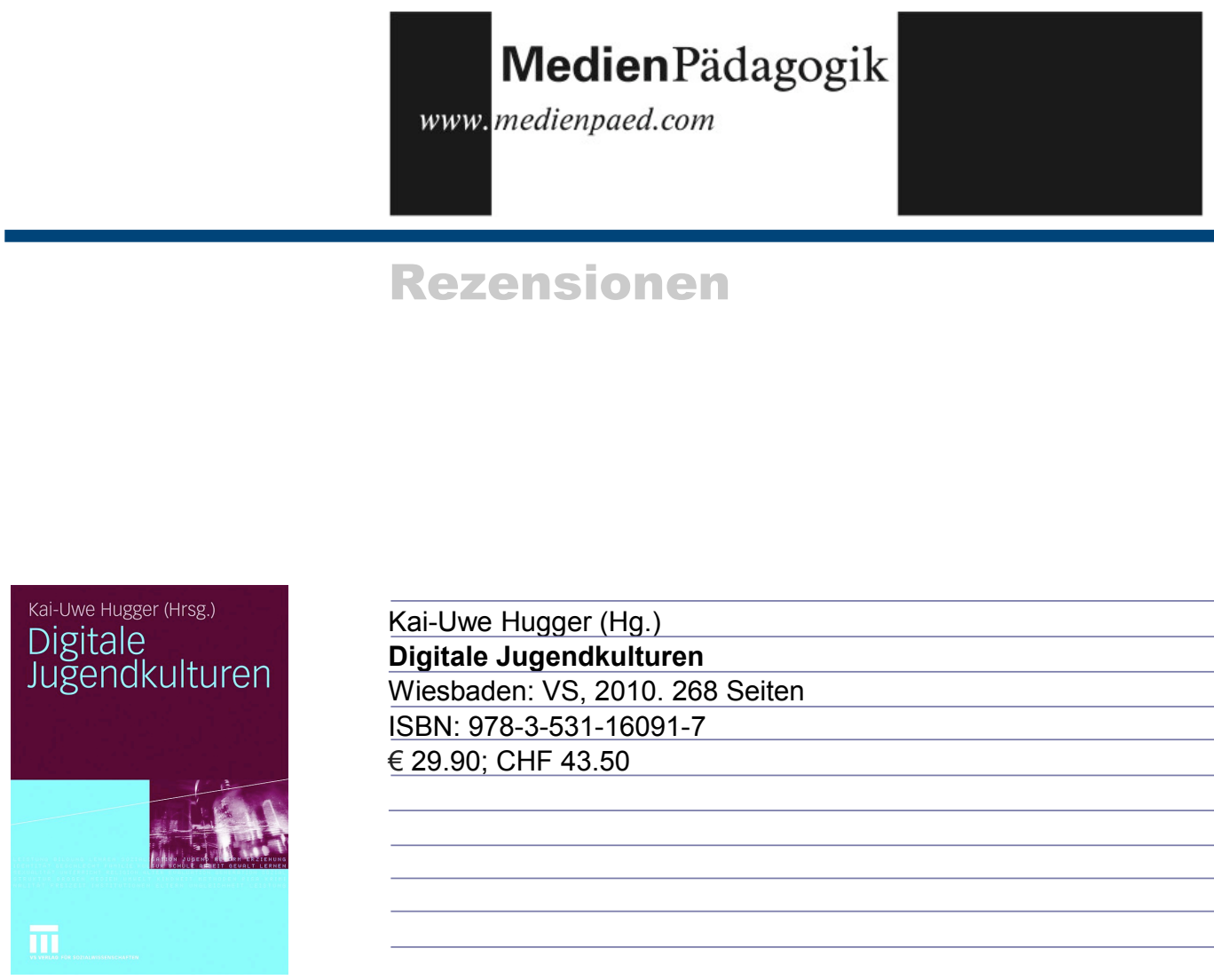

Kai-Uwe Hugger (Hg.)

Digitale Jugendkulturen

Wiesbaden: VS, 2010. 268 Seiten

ISBN: 978-3-531-16091-7

$€ 29.90 ;$ CHF 43.50

\title{
Jugendkulturen und medialer Wandel
}

Schon Bezeichnungen wie ,mediale Jugendkulturen' oder ,Medienjugend' suggerierten in der Vergangenheit eine Dichotomie, die kaum eine/r derjenigen, die sich ernsthaft damit beschäftigten, tatsächlich im Sinn hatte. Es wird nicht einfacher, wenn wir - zu Recht - noch ,analoge' und ,digitale' Medien gegenüberstellen, weil wir davon ausgehen, dass Technik-, Medien- und kultureller Wandel einander bedingen (z.B. Krotz 2009; Morley 2010) und deshalb mutmaßen, dass auch Jugendkulturen heute anders ,funktionieren' müss(t)en als in früheren Zeiten. Genau und nur in dieser einschließenden Perspektive ist aber der Titel des Sammelbandes zu verstehen, den der Kölner Medienpädagoge Kai-Uwe Hugger herausgegeben hat. Es geht um die Frage, wie junge Menschen im Kontext jugendkulturellerer Vergemeinschaftung bzw. Gesellung Medien gebrauchen, die kaum mehr anders als digital vorzustellen sind und die, denken wir an Computer, Internet und Social Web, nicht nur die Funktionspaletten analoger Medien integrieren (Medienkonvergenz), sondern auch auf der sozialen Praxis fußen, die sich rund um ihre analogen Vorläufer entwickelt hat. Erst im evolutionären Wechselspiel entsteht das Neue, das der Begriff «digital» markiert, wenn er mehr als eine technische Entwicklung bezeichnen soll.

Der Band «Digitale Jugendkulturen» versammelt Protagonist/innen der deutschsprachigen Jugend- und Medienforschung sowie der Pädagogik, die das Feld in insgesamt 15 Artikeln zu beleuchten suchen: im ersten Teil «Theoretische Facetten digitaler Jugendkulturen» eher übergreifend, im zweiten Teil «Digitale Angebote und jugendorientierte Aneignungsformen» eher anhand konkreter Szenen, Vergemeinschaftungs- und Gesellungsformen sowie medialer Angebote. Die Grenze zwischen den Sektionen verläuft fließend, da einige theoretisch orientierte Beiträge mit Empirie veranschaulichen und die meisten gegenstandsorientierten Beiträge eine theoretische Einordnung voranstellen.

In seiner Einleitung distanziert sich der Herausgeber ausdrücklich vom Leitmotiv einer «homogenisierenden Generationengestalt der ,Netz-Generati- 
on'», das er Diskursprägenden Darstellungen wie jenen von Kellner (1997), Tapscott (1998) und Palfrey/Gasser (2008) unterstellt. Vielmehr seien «die einzelnen jugendkulturellen Gesellungen vor allem in ihren partikularen Orientierungen» (14, Hervorh. i.O.) anzuerkennen. Gleichwohl sieht er in Anlehnung an Dieter Baacke «strukturelle Gemeinsamkeiten», wonach Jugendliche in Onlinewelten nach «Intensitäts-», "Ganzheits-» und «Subjektivitätserfahrungen» (15) suchen.

Der erste Teil des Bandes wird von Henrike Friedrichs und Uwe Sander eröffnet. Sie erinnern an die vergleichsweise junge Geschichte der Jugend als «Lebensabschnitt eigenen Rechts» (nach Werner Fuchs) (26) und betonen die Bedeutung der Medien, die sich im 20. Jahrhundert «zu einer mächtigen Sozialisationsinstanz entwickelt haben.» (28) Vom Panorama der Multioptionsgesellschaft ausgehend zeichnet Waldemar Vogelgesang das Bild einer Jugend, die «die Unsicherheiten und Risiken der vielgestaltigen Lebens- und Handlungssituationen produktiv» (39) annimmt und Jugend- und Medienkulturen ganz selbstverständlich als Bühnen der Selbstdarstellung und Identitätsarbeit nutzt. Birgit Richard beschäftigt sich mit der visuellen Selbstpräsentation in Onlineplattformen und deutet am Beispiel des Black Metal Starposen als Formen mimetischer Selbstdarstellung. Vornehmlich mit Blick auf statistische Analysen setzen sich Nadia Kutscher und Hans-Uwe Otto mit sozialen Ungleichheiten im Gebrauch digitaler Medien auseinander. Wilfried Ferchhoff und Kai-Uwe Hugger reflektieren in einem historisch angelegten Beitrag auf die Entstehung von Gleichaltrigengruppen in je unterschiedlichen Zeiten und diskutieren auf dieser Basis die These zunehmend de-lokalisierter und entgrenzter Gesellungsformen. Auf der Basis ihrer Theorie strukturaler Medienbildung beleuchten Benjamin Jörissen und Winfried Marotzki Orientierungs- und Bildungspotenziale, die in Praktiken des «Sharing» und «Social Networking» liegen, um mit Unbestimmtheiten in zunehmend posttraditional geprägten Gesellschaften umzugehen. Kai-Uwe Hugger und Ferdal Özcelik schließen den ersten Teil mit einem Beitrag zu intraethnischen Jugendgesellungen im Social Web ab, die sie in erster Linie als Ressource für die «Vergewisserung von ,prekärer' Mehrfachzugehörigkeit» (138) und als Ressource sozialer Anerkennung diskutieren.

Der zweite Teil des Bandes enthält ebenfalls sieben Artikel. Ziel ist wohl, mit den ausgewählten Themen die aktuelle Landschaft digitaler Medien und entsprechender Aneignungsformen nachzuzeichnen. Friederike von Gross stellt die (Musik-)Szene «Visual Kei» vor, die sich, ursprünglich in Japan entstanden, hierzulande ausschließlich über das Internet verbreitete und thematisch vor allem um die visuelle Selbstpräsentation und -inszenierung gruppiert. Die Beiträge von Sabina Misoch und Thorsten Quandt widmen sich Computerspielen: einmal mit Blick auf die Identifikation von Spieler/innen mit ihren Avataren und einmal überblickend als Synopse der Geschichte digitaler Spiele und medienpsychologischer Forschungsaspekte wie «Flow» oder Transfereffekte. Franz Josef Röll gibt einen Überblick zu Social Network Sites und diskutiert deren Potenziale und «Schattenseiten». Mit medienkon- 
vergenten Interaktionen beschäftigt sich Martina Schuegraf, die diese am Umgang Jugendlicher mit «Celebrities» veranschaulicht. Angela Tillmann stellt Mädchenräume im Internet vor und geht dabei nicht nur auf konkrete Angebote ein, sondern konturiert auch die Umrisse der wissenschaftlichen Auseinandersetzung der Aneignung des Internets durch junge Frauen. Der letzte Beitrag von Klaus Rummler versucht, die Mobiltelefonnutzung von Jugendlichen auf der Basis der quantitativen Daten der Verbraucheranalyse 2007/2008 in Beziehung mit alltagsästhetischen Milieuunterschieden zu setzen.

«Digitale Jugendkulturen» ist ein lesenwertes Buch. Zusammen sprechen die Beiträge ein breites Spektrum an Themen und Fragestellungen an und geben Einblicke in aktuelle mediale Praxen sowie Formen jugendkultureller Vergemeinschaftung und Gesellung. Das ist Sinn und Zweck eines solchen Sammelbandes. Zu Irritationen kann allerdings die ambivalente, häufig nicht explizierte Verwendung des Begriffs «Jugendkultur» führen. Wer erwartet, dass der Band, gleichsam als (medienfokussierte) Verlängerung und Aktualisierung von Arbeiten wie Baacke (1999), Müller-Bachmann (2002), Hitzler et al. (2005) oder Ferchhoff (2007) die Landschaften der diversifizierten Jugendszenen und -kulturen kartografiert, sucht eine solche Systematik vergebens. Mitunter stehen natürlich einzelne jener um ein spezifisches Thema gruppierten Vergemeinschaftungen (wie Black Metal, Visual Kei etc.) im Mittelpunkt; oft steht der Begriff aber auch einfach synonym für Formen kommunikativen Medienhandelns, die im Alltag von Jugendlichen bedeutsam sind.

Wie viele neue Einsichten die/der Einzelne aus der Lektüre mitnehmen kann, hängt freilich vom eigenen Vorwissen ab. Zwar sind die meisten Autor/innen bestrebt, die jüngsten medialen Entwicklungen, allen voran im Social Web, aufzunehmen und in ihrer Bedeutung zu reflektieren und Beiträge wie die von Birgit Richard zu «Bild-Ego» oder Friederike von Gross zu «Visual Kei» markieren tatsächlich neue Forschungsgegenstände. Jedoch kann der prägnante Titel darüber hinwegtäuschen, dass in weiten Teilen an bekannte Positionen der neueren Jugendmedienforschung und Jugendsoziologie angeschlossen wird. Gehen wir davon aus, dass sich der Wandel jugendkultureller und medialer Praxis als Prozess vollzieht, macht diese Kontinuität ja auch Sinn. Da wir mitten in der Entwicklung stecken und niemand heute mit Sicherheit sagen kann, welche Wege die Digitalisierung noch nehmen wird, kann auch dieses Buch keine letzten Antworten geben. Vielmehr geht es um eine Identifikation, welche der bestehenden Begründungsmuster noch tragen, welche womöglich zu ergänzen und welche auch zu verwerfen sind. In diese Richtung gehen die Beiträge von Friedrichs/Sander, Vogelgesang, Ferchhoff/Hugger und Jörissen/Marotzki. Mit Spannung ist in einigen Jahren eine Fortführung dieser Debatten als «Digitale Jugendkulturen: revisited» zu erwarten. 


\section{Literatur}

Baacke, Dieter. Jugend und Jugendkulturen. Darstellung und Deutung. 3., überarbeite Aufl. Weinheim u.a.: Juventa, 1999.

Ferchhoff, Wilfried. Jugend und Jugendkulturen im 21. Jahrhundert. Lebensformen und Lebensstile. Wiesbaden: VS, 2007.

Hitzler, Ronald; Bucher, Thomas; Niederbacher, Arne. Leben in Szenen. Formen jugendlicher Vergemeinschaftung heute. 2., aktualisierte Aufl. Wiesbaden: VS, 2005.

Kellner, Douglas. «Die erste Cybergeneration.» Kursbuch JugendKultur. Stile, Szenen und Identitäten vor der Jahrtausendwende. Hrsg. V. SPoKK. Mannheim: Bollmann, 1997. S. 310-316.

Krotz, Friedrich. «Mediatization: A concept to grasp media and societal change.» Mediatization: Concept, Changes, Conflicts. Ed. Knut Lundby. New York: Lang, 2009. 21-40.

Morley, David. «Kulturen, Wandel und Technologien. Ein kontextualistischer Ansatz.» Medienkultur im Wandel. Hrsg. v. Andreas Hepp, Marco Höhn und Jeffrey Wimmer. Konstanz: UVK, 2010. S. 41-54.

Müller-Bachmann, Eckart. Jugendkulturen revisited. Musik- und stilbezogene Vergemeinschaftungsformen (Post-)Adoleszenter im Modernisierungskontext. Münster u.a.: Lit, 2002.

Palfrey, John; Gasser, Urs. Generation Internet. Die Digital Natives: Wie sie leben, was sie denken, wie sie arbeiten. München: Hanser, 2008.

Tapscott, Dan. Net Kids. Die digitale Generation erobert Wirtschaft und Gesellschaft. Wiesbaden: Gabler, 1998. 